\title{
UN MODELO TEÓRICO DE REASIGNACIÓN DE RECURSOS ANTE UN SHOCK DE DEMANDA TURÍSTICA
}

\author{
Diego Rojas ${ }^{1}$
}

Recibido: 20/02/2014 Aprobado: 22/04/2014

\begin{abstract}
RESUMEN
Este trabajo analiza la relevancia de la actividad turística y su posible impacto sobre la economía costarricense, desde un punto de vista teórico. La ausencia de estudios formales cercanos a la realidad costarricense, y el uso de políticas e incentivos para la promoción del turismo hacen necesaria esta investigación.

Primero, se analizan los diferentes modelos que se han desarrollado en la literatura. Luego, se desarrolla el objetivo principal de esta investigación: construir un modelo que se acerca a las condiciones de la economía de Costa Rica. De forma específica, se tiene por objetivo discutir las siguientes preguntas: 1) ¿Cuál es el posible resultado sobre la producción interna, de largo plazo, de un aumento del gasto de los turistas?; 2) ¿En el largo plazo, qué sectores pueden ver aumentada su producción y que sectores pueden verla disminuida ante un aumento del gasto de los turistas? Y; 3) ¿Cuál es el comportamiento esperado de los precios relativos, de largo plazo, ante un aumento del gasto de los turistas?
\end{abstract}

PALABRAS CLAVES: TURISMO, BIENES NO TRANSABLES, BIENES TRANSABLES, APRECIACIÓN DEL TIPO DE CAMBIO.

\begin{abstract}
This paper analyzes the relevance and possible impact of tourism on Costa Rican economy, from a theoretical point of view. The absence of formal studies assuming conditions close to the Costa Rican economy and the wide application of incentives in this nation to promote tourism prove this research relevant.

First, the different models proposed in the literature are analyzed. Secondly, a model close to Costa Rican conditions is developed. The development of such model is the main objective of this paper. Specifically, the objective of this model is to shed some light on the following questions: 1) What is the possible impact on domestic production -in the long run- of an permanent increase in tourism demand for domestic goods?, 2) In the long run, what sectors might increase their production and what sectors might experience a decrease in production before a tourism demand increase? and, 3) What is the expected behavior of relative prices -in the long run- before an increase in tourism demand?
\end{abstract}

KEY WORDS: TOURISM, TRADABLES, NON-TRADABLES, EXCHANGE RATE APPRECIATION.

1 Banco Mundial, Oficina del Economista en Jefe para América Latina y el Caribe, USA, Código Postal WASHINGTON-DC, 20015; lrojasalvarado@worldbank.org.

Los resultados, interpretaciones y conclusiones son completamente del autor y no representan aquellas de ninguna de las instituciones a las que el autor está afiliado. 


\section{INTRODUCCIÓN}

La economía costarricense desde la década de los ochenta ha sufrido cambios importantes en su sector externo. Por ejemplo, se aceleró el proceso de desgravación arancelaria, se incrementó la inversión extranjera directa y aumentó el turismo. Respecto a este último, se han tomado medidas específicas para incentivarlo. Ya desde 1955, por medio de la Ley Orgánica No 1917, se crea el Instituto Costarricense de Turismo (ICT) cuya misión es "promover el desarrollo turístico integral con el fin de mejorar el nivel de vida de los costarricenses..." y tiene como finalidad principal incrementar el turismo en el país.

Más recientemente, la Asamblea Legislativa decreta la Ley de Incentivos para el Desarrollo Turístico. Publicada en La Gaceta No.143 del 30 de julio de 1985, dicha ley declara de utilidad pública la industria del turismo, $y$ tiene por objeto "establecer un proceso acelerado y racional de desarrollo de la actividad turística costarricense, para lo cual se establecen los incentivos y beneficios que se otorgarán como estímulo para la realización de programas y proyectos importantes de dicha actividad". Dentro de los incentivos otorgados por dicha ley destacan: la exención de todo tributo y sobretasas que se apliquen a la importación o compra local de los artículos indispensables para el funcionamiento o instalación de empresas nuevas; la concesión de patentes municipales que requieran dichas empresas y la exoneración del impuesto territorial hasta por un periodo de seis años. Dichos incentivos muestran el interés de los legisladores por promover las actividades ligadas al turismo en nuestro país, lo cual presupone que dicho sector beneficiaría a la economía.

Por otra parte -independientemente de si es resultado o no de las políticas aplicadas por el ICT- la actividad turística ha crecido en las últimas décadas, para convertirse en un elemento de importancia dentro de la economía costarricense ${ }^{2}$. Así por ejemplo, el número de turistas que ingresan a Costa Rica, pasó de representar el 10\% de la población en 1986, al 50\% de la población en el 2012. Más aun, el número de visitantes extranjeros que ingresó al país entre 1986 y 2012 se multiplicó por 9, con una tasa de crecimiento promedio anual del 9\%. Adicionalmente el ingreso de divisas por concepto de turismo representaba el 6,37\% del consumo final de los hogares en 1991, y dicho porcentaje aumentó hasta un $11,87 \%$ en 2005. Además desde el 2000, el ingreso por divisas constituye el $20 \%$ del total de exportaciones, posicionando al turismo como uno de los principales "productos de exportación" del país.

Estos datos evidencian la importancia que ha tomado el turismo dentro de la actividad económica de Costa Rica, mientras que la Ley No 6990 muestra el compromiso por parte del Gobierno en incentivar dicho tipo de actividades; pero esta evolución del sector turístico no ha estado acompañada de un análisis riguroso de su impacto en la economía. Los argumentos para promover el turismo se han basado esencialmente en "la generación de empleo", y el posible aumento en la producción (ingreso); sin embargo esta motivación podría ser falaz, al menos a nivel teórico, como se observa en los modelos formales desarrollados en la literatura económica hasta el momento.

En el presente trabajo se desarrolla un modelo teórico para analizar las posibles repercusiones del aumento del turismo en Costa Rica. Para esto se divide el país en regiones que producen bienes transables $y$ un bien no transable que es demandado por los turistas y es particular a la región del país. En el modelo se argumenta que si todos los factores son móviles a nivel internacional, y el único factor no móvil es la tierra, tendríamos regiones dedicadas exclusivamente al turismo y otras a la producción del bien transable. En este caso, si el turismo aumenta la demanda de los bienes no transables en esta región, aumentará la contratación en la región, el precio de la tierra, la producción y por tanto el ingreso.

2 En Rojas (2009) y Yong et al (2009) se pueden encontrar mayores detalles sobre la importancia del turismo en la economía costarricense. 
Las conclusiones se modifican si existe un factor no móvil a nivel internacional que es compartido entre las regiones, por ejemplo el trabajo calificado. Un aumento de la demanda por el producto no transable de cierta región, elevaría la demanda por todos los factores de producción en este sector. El primer efecto que se podría esperar es un desplazamiento de la producción de transables en la misma región. Por ejemplo, el aumento en la actividad turística se daría a costa de una disminución de la actividad agrícola. Además, con el crecimiento de la demanda por el factor de producción que no se puede contratar del extranjero, se daría un aumento de su precio y un desplazamiento desde las demás zonas. Esto provoca que disminuya la oferta en las otras regiones, incrementando el precio de todos los bienes no transables en el país - y no solamente el precio del no transable demandado por los turistas.

Así el turismo puede aumentar el ingreso en ciertas zonas y a ciertos factores de producción, pero por el otro lado, puede elevar el precio de los bienes no transables en todo el país. El resultado sobre el bienestar depende de cuál efecto sea mayor: el efecto positivo sobre el ingreso o el efecto negativo sobre el precio. No obstante, no se conciben situaciones en las cuales el efecto negativo sobre el precio pueda afectar en forma importante a ciertos sectores. Por ejemplo, los trabajadores no calificados con un salario mínimo que se encuentra fijo -i.e, y no aumenta con la llegada de los turistas, por existir inmigración de este tipo de mano de obra- pero que enfrentan el incremento del precio de los transables. En general se pueden esperar efectos redistributivos del ingreso, en donde el sector que produce para los turistas gana del aumento del ingreso, pero los otros sectores se ven perjudicados por el alza en el precio de los bienes no transables. Además se tendría una nueva asignación en la producción, donde los sectores transables son desplazados por los sectores no transables, demandados por los turistas.

En este trabajo, entonces, se evalúa la relevancia de la actividad turística y su posible impacto sobre la economía. La ausencia de estudios formales, cercanos a la realidad costarricense, y la utilización de políticas e incentivos para su promoción hacen necesaria esta investigación. Ante este panorama se propone analizar los diferentes modelos que se han desarrollado para estudiar el turismo y a la vez construir un modelo que se acerque más a las condiciones de la economía costarricense. La construcción de dicho modelo es el principal propósito de esta investigación. De forma específica, se tiene por objetivo discutir las siguientes preguntas: 1) ¿Cuál es el posible resultado sobre la producción interna, de largo plazo, de un aumento del gasto de los turistas en una economía como la costarricense?; 2) ¿En el largo plazo, qué sectores pueden ver aumentada su producción y que sectores pueden verla disminuida ante un aumento del gasto de los turistas en una economía como la costarricense? y; 3) ¿Cuál es el posible resultado sobre los precios relativos, de largo plazo, de un aumento del gasto de los turistas en una economía como la costarricense?

Para responder a estas preguntas, en la siguiente sección se presentan las principales características de los modelos desarrollados hasta el momento en la literatura económica. En la sección siguiente desarrolla el modelo final, que toma en cuenta la diferenciación de los productos, que consumen los turistas, dependiendo de la región del país que visiten. Por último se presentan las conclusiones generales.

\section{Modelos en la literatura}

En la literatura sobre comercio de servicios se distinguen las exportaciones de turismo de las exportaciones de bienes, porque el turista consume principalmente bienes no transables del país que visita. Según Copeland (1991), a diferencia de las exportaciones de bienes, “...los turistas deben visitar el país doméstico para comprar y consumir bienes y servicios turísticos (...) como resultado bienes que normalmente son no transables, como servicios de restaurante, se convierten parcialmente en transables con la presencia del turismo". 
Por lo anterior, el turismo es normalmente analizado como una perturbación de demanda sobre el sector no transable de la economía. Normalmente se espera que esta mayor demanda externa por un bien nacional se refleje en un aumento del bienestar, pero los diferentes trabajos teóricos sobre el turismo enfatizan la posibilidad de una disminución en el bienestar al incrementarse dicha actividad.

Por ejemplo, el trabajo de Chao et al (2005) concluye que si el sector transable es fuertemente intensivo en capital, el aumento del turismo podría generar una disminución del bienestar. El rasgo principal de dicho modelo es que el Gobierno es el encargado de fijar el salario, lo cual genera desempleo. El aumento en el turismo en este modelo, aumenta la demanda por los bienes no transables $y$ con esto su precio relativo, esto a su vez aumenta el empleo pero (por Rybczynski) disminuye el nivel de capital (de estado estacionario). Si dicha disminución en el capital es lo suficientemente grande (es decir, si el sector transable que se contrae es muy intensivo en dicho factor), se daría una disminución en el bienestar de los residentes.

Por otra parte, el modelo de Nowak et al (2003) introduce rendimientos crecientes a escala en el análisis. Su conclusión es que el aumento del turismo puede aumentar la pérdida de bienestar generada por existencia de un sector con rendimientos crecientes.

La característica particular del modelo de Hazari y Nowak (2003) es el supuesto de poder monopólico en la producción del bien consumido por los turistas. En su contexto de dos países $y$ poder monopólico, la principal conclusión del modelo es que el equilibrio encontrado (de libre comercio) es subóptimo, lo cual justifica la imposición tributaria al turismo. Además, muestran como un aumento en la demanda por parte de los turistas puede disminuir el bienestar de los residentes vía una disminución de los términos de intercambio.

El modelo de Copeland (1991) supone que en la economía se producen tres bienes finales: agricultura y manufactura, los cuales son transables; $y$ servicios, los cuales son no transables. Además asume una economía pequeña con factores que son ofrecidos de forma completamente inelástica. Con estos supuestos concluye que el aumento del turismo solo mejora el bienestar de los residentes si se eleva el precio relativo de los no transables, es decir, si se da una mejora en los términos de intercambio; pero esto puede ser atenuado si los factores de producción son móviles. Una estimación de este modelo para el caso de Costa Rica se encuentra en Rojas (2008), el cual se basa en la metodología utilizada en Ulate y Rojas (2006) y propuesta inicialmente en Porto (2003).

Un mayor detalle de estos modelos se encuentra en Rojas (2008), pero en general, la posible pérdida de bienestar de un aumento del turismo surge del desplazamiento de la producción de algunos sectores de la economía, cuando aumenta la producción en otros sectores. Dicha observación no solamente ha sido discutida a nivel teórico. Existe evidencia empírica que muestra como, en ciertos casos, el desarrollo del turismo va en detrimento de algún otro sector, especialmente la agricultura. Como ejemplos tenemos los trabajos de Tyrakowski (1986) para la costa mediterránea de España, Bryden (1973) y Weaver (1988) para los países del Caribe, entre otros.

Por otra parte, excluyendo el modelo de Copeland (1991), se puede notar que los demás modelos no son aplicables a la economía costarricense; ya que suponen que los factores no son móviles a nivel internacional, además de alguna distorsión que provoca la disminución en el bienestar. Algunas de estas distorsiones no pueden justificarse para el caso costarricense, pero en especial, el país presenta apertura para la libre movilidad internacional tanto del capital como del trabajo. En particular, no se pueden identificar restricciones importantes para importar o exportar bienes de capital o para comprar o vender activos externos.

Además, el fenómeno de la migración ha tomado importancia en el país, por lo que tampoco se debe descartar la movilidad del trabajo. Esto se concluye de trabajos como el de Barquero y Vargas (2004), en donde se plantea que la población inmigrante aumentó en Costa Rica especialmente en la década de los noventa. Estiman que "entre 1984 y 2000 la población nacida en el extranjero pasó de casi 90.000 a cerca de los 300.000 residentes en el país, de los cuales 226 mil correspondieron a la 
población de origen nicaragüense. En términos relativos este crecimiento implicó que el porcentaje de población extranjera se duplicara, al pasar de 4 a 8 por ciento del total de población de Costa Rica en el 2000, aumentando los nicaragüenses a tres cuartas partes del total de extranjeros". Por último, concluyen que "la migración nicaragüense es fundamentalmente laboral".

Dicho trabajo muestra que la importancia de la inmigración en nuestro país ha crecido a partir de la década de los noventa, en especial de población nicaragüense y fundamentalmente se debe a la búsqueda de empleo, lo cual resulta relevante para este trabajo.

El modelo de Copeland (1991) parece entonces ser la base para este análisis; pero respecto a este se identificó una particularidad muy importante, a pesar de que supone libre movilidad de ciertos factores, no incluye un activo financiero externo con el cual el país puede ahorrar o endeudarse con el resto del mundo. En el desarrollo de este trabajo, se incluye dicho activo; además se incorpora la posibilidad de bienes diferenciados por cada región del país, lo cual parece adecuado para analizar el caso de Costa Rica, como se argumentará en la próxima sección. Estos cambios marcar un distanciamiento de todos los modelos anteriores y presentan la principal contribución del artículo.

Con estas consideraciones, la siguiente sección desarrolla un modelo base para analizar el efecto de la actividad turística sobre el bienestar en Costa Rica.

\section{Modelo con productos no transables diferenciados por región}

El objetivo de esta sección es desarrollar un modelo que tome en cuenta la posibilidad de bienes no transables diferenciados por región, lo cual se considera adecuado para analizar la economía costarricense. Los turistas tienden a visitar Costa Rica por ciertas características que posee el país, como su flora, fauna y playas. Estos atributos se encuentran en diferentes zonas, por lo que es de esperar, que los bienes consumidos por los turistas (que incorporan el consumo de estas características) sean diferentes en cada región de Costa Rica. Nótese que, aunque los turistas no perciban como diferente cada región de Costa Rica, es suficiente que los productores sí lo hagan, para poder modelar un producto por región.

Así, se supone que cada región produce un bien diferente, o de forma inversa, se define la región como aquella en la que se produce un bien, considerado distinto. Esta definición es lo suficientemente flexible como para que el modelo termine analizando casos en los que muchos productos son considerados diferentes, o en donde todas las regiones son iguales, pues los bienes no se diferencian, por lo que tendríamos una sola región y un solo bien no transable.

Se observó que uno de los incentivos de los turistas para visitar el país son sus atractivos naturales, como bosques y playas; los cuales se ubican en diferentes zonas del territorio, además las actividades que se realizan pueden diferir según sea el atractivo ${ }^{3}$. Por esto se presenta un modelo que abre a la posibilidad de bienes diferenciados dependiendo de la región del país. Dicho caso no ha sido analizado y se considera que es de gran importancia en Costa Rica, en donde el turismo tiene como base las "bellezas" naturales, las cuales dependen de la región particular del país.

Se supone que la economía es pequeña y abierta. En dicha economía se produce un bien transable, que se denota con el subíndice $T$. Este bien transable es utilizado como numerario. Se tienen 3 factores de producción: capital $(K)$, trabajo $(L)$ y tierra $(S)$. El capital es homogéneo y libre de moverse tanto entre sectores como entre países. Una unidad de capital puede transformarse en una de transable a cero costo.

El trabajo es de dos tipos no calificado $L_{n}$ y calificado $L_{c}$. El trabajo no calificado puede moverse libremente entre sectores y países, mientras que el trabajo calificado solo puede moverse entre sectores. Adicionalmente, el país se divide en $N$ regiones. Cada una de estas posee un tipo de

3 Dicha información se deriva de las encuestas realizadas por el ICT a los turistas. Según estas encuestas, la gran mayoría de turistas visitan el país por motivos de ocio y entre las actividades más realizadas se encuentran sol y playa, caminatas por senderos, observación de flora $y$ fauna; $y$ la visita a volcanes. 
tierra diferente (montaña, playa, bosque, etc.) y a su vez características particulares. Estas diferencias se asocian al factor tierra y en cada tipo de tierra se produce un bien no transable diferente. Se asume, entonces, que en cada uno de los tipos de tierra, se produce un bien diferente (y solo uno). Adicionalmente en cada región se puede producir el bien transable $T$. Cada uno de los bienes diferenciados no es transable. De cada tipo $i$ de tierra se cuenta con una dotación $\bar{S}_{i}$. Adicionalmente se supone que dentro de cada región los mercados se comportan de manera competitiva.

\section{Consumo}

Se asume un agente representativo que deriva utilidad del consumo del bien transable $T$ y de los bienes no transables. La función de utilidad de dicho agente es representada por:

$$
U=\sum_{t=t_{0}}^{\infty} \beta^{t-t_{0}}\left[\gamma_{T} \ln \left(C_{T, t}\right)+\sum_{i=1}^{N} \gamma_{i} \ln \left(C_{i . t}\right)\right]
$$

En donde $C_{T}$ es el consumo total del bien $T$, $C_{i}$ es el consumo del bien (no transable) $i, \beta$ es un factor subjetivo de descuento, $0<\gamma_{j}<1$ y el subíndice $t$ simboliza el tiempo. El agente representativo maximiza la función de utilidad sujeto a la restricción

$$
B_{t+1}-B_{t}+\sum_{i=1}^{N} I_{i, t}+I_{T, t}=r B_{t}+Y_{T, t}-C_{T, t}+\sum_{i=1}^{N} p_{i, t} Y_{i, t}-\sum_{i=1}^{N} p_{i, t} C_{i, t}
$$

En donde $B_{t}$ representan el acervo del (único) activo financiero al inicio del periodo $t$, $I_{j}=K_{t+1, j}-K_{t, j}$ es la inversión en el sector $j, Y_{T, t}$ es la producción total del bien $T$, $Y_{i}$ la producción del bien $i$ y $p_{i}$ su precio.

Suponiendo que $\beta(1+r)=1$, las condiciones de primer orden de este problema implicarían que

$$
C_{T, t}=C_{T, t+1}:=C_{T}
$$

es decir, el consumo del bien transable se suaviza en el tiempo.

Además

$$
C_{i, t}=\left(\frac{\gamma_{i}}{\gamma_{T}}\right) \frac{C_{T}}{p_{i, t}}
$$

el precio relativo iguala a la tasa marginal de sustitución.

Adicionalmente, se asume que la demanda de los turistas $V_{i}$ por el bien $i$ es similar a la de los residentes

$$
V_{i, t}=\frac{V_{i}^{*}}{p_{i, t}}
$$

en donde $V_{i}^{*}>0$ es constante.

Como cada uno de los bienes diferenciados no es transable, su precio se determina dentro de la economía. Es decir, la cantidad consumida debe igualar a la producida, $Y_{i, t}=C_{i, t}+V_{i, t}$. Utilizando (1) se tiene entonces que 


$$
Y_{i, t}=\frac{\gamma_{i}}{\gamma_{T}} \frac{C_{T}}{p_{i, t}}+\frac{V_{i}^{*}}{p_{i, t}}
$$

de lo cual se obtiene que

$$
p_{i, t}=\frac{\left(\gamma_{i} / \gamma_{T}\right) C_{T}+V_{i}^{*}}{Y_{i, t}}
$$

\section{Producción}

El productor tiene que decidir entre cuánto ocupar de la tierra, en la zona $i$, para la producción del bien $T y$ del bien $i$. Se supone que los sectores $i$ utilizan como factores de producción el trabajo calificado, no calificado, capital y la tierra. El $L_{n}$ y $K$ son móviles a nivel internacional, por lo que el salario $w_{n} y$ la tasa de interés $r$ están dadas, a su nivel internacional ${ }^{4}$.

La producción de cada uno de estos bienes se representa por una función de rendimientos constantes a escala, tipo Cobb-Douglas

$$
Y_{i, t}=A_{i} L_{n, i, t}^{\theta_{i}} L_{c, i, t}^{\delta_{i}} S_{i, t}^{\psi_{i}} K_{i, t}^{\sigma_{i}}
$$

en donde se supone que $\theta_{i}+\delta_{i}+\psi_{i}+\sigma_{i}=1$. Los productores buscan maximizar el valor descontado de sus beneficios. Este problema de maximización para la variedad $i$ es:

$$
\operatorname{Max} \sum_{t=t_{0}}^{\infty}\left(\frac{1}{1+r}\right)^{t-t_{0}}\left[p_{i, t} Y_{i, t}-w_{n} L_{n, i, t}-w_{c} L_{c, i, t}-p_{s, i, t}\left(S_{i, t+1}-S_{i, t}\right)-\left(K_{i, t+1}-K_{i, t}\right)\right]
$$

Como se supone un comportamiento competitivo, los productores toman $p_{i}$ como dado, $y$ deciden sobre $\mathrm{L}_{\mathrm{n}, \mathrm{i}, \mathrm{t}}, \mathrm{L}_{\mathrm{c}, \mathrm{i}, \mathrm{t}}, \mathrm{K}_{\mathrm{i}, \mathrm{t}+1}$ y $\mathrm{S}_{\mathrm{i}, \mathrm{t}+1}$. Las condiciones de primer orden de este problema serían

$$
\begin{aligned}
& p_{i, t} \delta_{i} A_{i} s_{i, t}^{\psi_{i}} k_{i, t}^{\sigma_{i}} l_{c, i, t}^{\delta_{i}-1}=w_{c} \\
& p_{i, t} \sigma_{i} A_{i} s_{i, t}^{\psi_{i}} k_{i, t}^{\sigma_{i}-1} l_{c, i, t}^{\delta_{i}}=r \\
& p_{i, t} \theta_{i} A_{i} s_{i, t}^{\psi_{i}} k_{i, t}^{\sigma_{i}} l_{c, i, t}^{\delta_{i}}=w_{n} \\
& p_{s, i, t}=\left(\frac{1}{1+r}\right)\left(p_{s, i, t+1}+p_{i, t} \psi_{i} A_{i} s_{i, t+1}^{\psi_{i}-1} k_{i, t}^{\sigma_{i}} l_{c, i, t+1}^{\delta_{i}}\right)
\end{aligned}
$$

En donde $l_{c}=L_{c} / L_{n}, s=S / L_{n}, k=K / L_{n}$ son las relaciones de los factores respecto al trabajo no calificado y se rezagó la condición respecto al capital un periodo.

Las relaciones (5) y (7) muestran que el valor del producto marginal del trabajo debe igualar al salario. La relación (6) evidencia que el valor del producto marginal del capital iguala a la tasa de interés real $r$. Y la relación (8) demuestra que, en el óptimo, el valor presente neto de adquirir una unidad adicional de tierra debe ser cero.

Combinando (6) y (7) se obtiene que:

$$
k_{i, t}=\frac{\sigma_{i}}{\theta_{i}} \frac{w_{n}}{r}
$$

$4 \quad$ En el caso del trabajo no calificado, puede interpretarse como que el salario se mantiene al nivel del salario mínimo. 
Como $r$ y $w_{n}$ están dados a nivel internacional, la relación anterior fija la relación capitaltrabajo no calificado para cada sector $i$. Utilizando (5) y (7) se obtiene que la relación entre trabajo calificado y no calificado depende del salario relativo del trabajo no calificado.

$$
l_{c, i, t}=\frac{\delta_{i}}{\theta_{i}} \frac{w_{n}}{w_{c}}
$$

Utilizando (5) , (9) y (10) se obtiene

$$
S_{i, t}=\left(A_{i} p_{i, t}\right)^{-\frac{1}{\psi_{i}}}\left(\frac{w_{n}}{\theta_{i}}\right)^{\frac{\theta_{i}+\psi_{i}}{\psi_{i}}}\left(\frac{r}{\theta_{i}}\right)^{\frac{\sigma_{i}}{\psi_{i}}}\left(\frac{w_{c}}{\delta_{i}}\right)^{\frac{\delta_{i}}{\psi_{i}}}
$$

Además utilizando $s_{i, t}=s_{i, t+1}$, (7) y (8) se tiene que

$$
p_{s, i, t}=\left(\frac{1}{1+r}\right)\left(p_{s, i, t+1}+\frac{\psi_{i}}{\theta_{i}} \frac{w_{n}}{s_{i, t}}\right)
$$

Se asume que en el sector $T$ solamente se utilizan como factores de producción $L_{n}$ y $S$. Con supuestos similares respecto a la función de producción, el problema de maximización de beneficios (para la región $i$ de tierra) sería:

$$
\operatorname{Max} \sum_{t=t_{0}}^{\infty}\left(\frac{1}{1+r}\right)^{t-t_{0}}\left[A_{T, i} L_{n, T, t}^{\theta_{T}} S_{T, i . t}^{\psi_{T}}-w_{n} L_{n, T, t}-p_{s, i, t}\left(S_{T, i, t+1}-S_{T, i, t}\right)\right]
$$

en donde $\psi_{T}=1-\theta_{T}$.

Las condiciones de primer orden serían

$$
\begin{aligned}
& s_{T, i, t}=\left(\frac{w_{n}}{\theta_{T} A_{T, i}}\right)^{\frac{1}{\psi_{T}}} \\
& p_{s, i, t}=\left(\frac{1}{1+r}\right)\left(p_{s, i, t+1}+H_{i}\right)
\end{aligned}
$$

en donde

$$
H_{i}=\psi_{T} A_{T, i}^{\frac{1}{\psi_{T}}}\left(\frac{\theta_{T}}{w_{n}}\right)^{\frac{\theta_{T}}{\psi_{T}}}
$$

Se debe notar que las relaciones (12) y (14) dicen que el valor presente neto de la tierra es cero. Estas relaciones están funcionando como una demanda por tierra; con lo cual reflejan la valoración que hacen los productores por la tierra en sus diferentes usos.

Imponiendo la condición de transversalidad $\lim _{T \rightarrow \infty} \frac{p_{s, i, t+T}}{(1+r)^{T}}=0$, en general, dicha valoración por la tierra estaría definida por:

$$
p_{s, i, t}=\sum_{s=t+1}^{\infty}\left(\frac{1}{1+r}\right)^{s-t} \text { (Valor del producto marginal) }
$$

En el presente modelo, se tiene que el valor del producto marginal de la tierra en el sector $T$ está dado por la constante $H_{i}$. Entonces, se define el precio de la tierra por

$$
r p_{s, i}=H_{i}
$$


Nótese que el sector $T$ está fijando el precio de la tierra (en este caso particular) y al no cambiar ninguno de los determinantes de $H_{i}$, dicho precio no varía en el modelo. Combinando (15) y (12) se obtiene que

$$
S_{i, t}=\frac{\psi_{i}}{\theta_{i}} \frac{w_{n}}{H_{i}}
$$

Esta relación fija la relación tierra-trabajo no calificado para cada sector $i$, mientras que la relación capital-trabajo no calificado está determinada por (9). Estas dos se mantienen constantes. Por su parte la relación entre trabajos $l_{c}$ depende del salario del trabajo calificado $w_{c}$, el cual al no ser móvil internacionalmente, tiene que determinarse dentro de la economía.

Combinando (16) con (11) se obtiene

$$
w_{c}=\delta_{i}\left[\left(\frac{\theta_{i}}{w_{n}}\right)^{\theta_{i}}\left(\frac{\psi_{i}}{H_{i}}\right)^{\psi_{i}}\left(\frac{\sigma_{i}}{r}\right)^{\sigma_{i}} A_{i} p_{i}\right]^{\frac{1}{\delta_{i}}}
$$

La anterior deja dependiendo el $w_{c}$ - que están dispuestos a pagar los productores de $i$ - del precio del bien final $i$.

Antes de analizar la determinación del precio del bien, supongamos por un momento que se da un aumento del turismo en uno de los sectores $i$ y que este incremento se refleja en un alza del precio $p_{i}$. Con lo que se ha desarrollado, se tiene un aumento en la demanda de todos los factores en el sector $i$, lo cual tendería a desplazar tierra hacia este sector estrujando al sector $T$. Adicionalmente se contrataría más tierra, capital y trabajo no calificado en este sector $i$ para atender esa mayor demanda.

Por el momento se sabe lo que sucedería con el trabajo calificado. De las relaciones (17) y (10) se tiene un aumento del salario y una disminución en la relación $L_{c} / L_{N^{*}}$. Esta disminución podría darse incluso ante un aumento en $L_{c}$, siempre y cuando este sea menor al cambio que se da en $L_{N}$. Si este fuera el caso, no solamente se desplaza el sector $T$, sino también los demás bienes no transables, al demandarse más trabajo calificado en un sector $i$ particular. Como lo que falta por analizar -por el lado la producción- es el factor $L_{c}$, se puede omitir para analizar un caso particular que es de interés, el cual se presenta en la siguiente sección.

\section{Modelo sin trabajo calificado}

Se asume que el modelo se comporta como se ha venido analizando, solamente se elimina el trabajo calificado $L_{c}$; de tal manera que cada uno de los sectores $i$ utiliza para producir únicamente capital, tierra y trabajo no calificado.

Nuevamente las relaciones (9) y (12) serían válidas, pero ahora se tendría que

$$
s_{i, t}=\left(A_{i} p_{i}\right)^{-\frac{1}{\psi_{i}}}\left(\frac{w}{\theta_{i}}\right)^{\frac{1-\sigma_{i}}{\psi_{i}}}\left(\frac{r}{\sigma_{i}}\right)^{\frac{\sigma_{i}}{\psi_{i}}}
$$

Entonces (12) se expresa como

$$
p_{s, i, t}=\left(\frac{1}{1+r}\right)\left(p_{s, i, t+1}+G\left(p_{i, t}\right)\right)
$$

en donde $G($.$) depende de forma directa del precio. Como en el estado estacionario p_{s, i, t+1}=p_{s, i, t}$, se tiene que

$$
r p_{s, i, t}=G\left(p_{i}, t\right)
$$

lo cual es similar a iterar la ecuación anterior, suponer $p_{i}$ invariante en el tiempo y utilizar la condición de transversalidad 


$$
\lim _{T \rightarrow \infty}\left(\frac{p_{s, i, t+T}}{(1+r)^{T}}\right)=0
$$

Como se mencionaba anteriormente esta relación da la valoración que hace el productor de $i$ de la tierra. Al no variar los supuestos sobre el sector $T$ la ecuación (15) es válida. Entonces, ¿Cuál sería el precio de la tierra? Note que tanto (18) como la condición (14) representan el valor presente de este activo. Dicho valor presente iguala el valor en el periodo siguiente más el (valor del) producto marginal de la tierra en cada sector. Comparando estas dos relaciones, se tiene que el sector $i$ valora más la tierra de la región $i$ cuando:

$$
G\left(p_{i}\right)>H_{i}
$$

Es decir, cuando el valor del producto marginal de la tierra es mayor en este sector que en el sector $T$. Es de esperar que el precio esté entre las dos valoraciones, que en el estado estacionario están dadas por $G\left(p_{i}\right) / r$ y $H_{i} / r$. Entonces, se tiene que para los sectores donde se cumple (19), la valoración es mayor en el sector $i$ que en el $T$. Por lo tanto, sería de esperar que los productores de $i$ se "adueñaran" de toda la tierra en las zonas donde se cumple esta condición. De forma contraria, se esperaría que toda la tierra se use en la producción del bien $T$ cuando la relación (19) no se cumpla. En este caso, se tienen zonas totalmente dedicadas a la producción del bien no transable y otras dedicadas exclusivamente a la producción del bien transable $T$.

Si se supone un aumento de la demanda de turistas para todos los sectores $i^{5}$, aumentaría los precios $p_{i} y$ esto provoca un aumento en el valor del producto marginal tanto del trabajo como del capital en los sectores $i$, esto induce a que aumente la cantidad contratada de estos factores en los no transables $i$.

Para los bienes $i$ que operaban (es decir que tenía la tierra $i$ contratada), dicho aumento en el precio eleva el valor del producto marginal de la tierra, pero al estar ya toda contratada en dicho sector, se da un incremento en su rendimiento. Lo anterior implica una disminución en la relación tierra-trabajo en estos sectores.

Adicionalmente, en los casos donde la relación (19) no se cumplía, caben dos posibilidades: que el aumento en el precio $p_{i}$ sea lo suficientemente alto como para que se desplace al sector $T$ de esas regiones; o que el aumento no sea lo suficientemente alto y se siga produciendo $T$ en dichas regiones. Para los bienes que cumplen la primera alternativa se tendría una descenso en la producción de $T$ (al ser desplazada), mientras que en los otros sectores no se vería afectada. Estos resultados dependen del valor de la tierra, el cual (en el modelo) queda determinado principalmente por $p_{i}$ $y$ por las productividades $A_{j}$ de cada sector.

Sería de esperar entonces, que el aumento en la demanda de los turistas no modifique la producción de $T$ en ciertas zonas (es decir con una productividad $A_{T}$, muy alta), pero que en ciertas regiones se vea desplazada dicha actividad. Note que la producción de $T$ en la región $i$ (que se denota por $Y_{T, i}$ ) puede expresarse como

$$
Y_{T, i}=\frac{H_{i}}{\psi_{T}} \bar{S}_{l}
$$

en donde es el total de tierra en la región $i$. De forma similar $p_{i} Y_{i}$ puede representarse por

$$
p_{i} Y_{i}=\frac{G\left(p_{i}\right)}{\psi_{i}} \bar{S}_{\imath}
$$

\footnotetext{
$5 \quad$ Se supone un aumento en todos los sectores, ya que un incremento de la demanda por solo uno de los bienes $i$ no tendría efecto sobre los otros bienes no transables, esto debido a que los únicos factores que comparten son móviles internacionalmente, así que un sector puede aumentar o disminuir la contratación de estos sin tener que provocar cambios en la cantidad de los otros sectores, pues simplemente pueden importarlo o exportarlo.
} 
De las anteriores se obtiene que $p_{i} Y_{i}>Y_{T, i}$ si

$$
G\left(p_{i}\right)>\frac{\psi_{i}}{\psi_{T}} H_{i}
$$

Entonces, si se cumple (22) se tiene que: cuando se produce $\mathrm{Y}_{\mathrm{i}}$ en la región $i$, dicha producción es mayor a la que se obtendría si se produjera $Y_{T}$. Entonces, si el aumento en el gasto de los turistas produce que se desplace la producción en cierta región de $Y_{T}$ hacia $Y_{i}$, se tendría un aumento en el producto de dicha región.

En general, este caso donde se excluye el trabajo calificado, es de particular interés ya que estaría denotando regiones exclusivamente dedicadas a la producción de no transables -es decir, dedicadas exclusivamente al turismo- $y$ otras exclusivamente dedicadas a la actividad transable. En el caso en el que se incluye el trabajo calificado, esto no sucede ya que el trabajo calificado pone un tope a la contratación de los demás factores.

\section{Modelo con trabajo calificado}

Retomando el modelo principal, faltaba determinar el salario del trabajo calificado. Para esto, primero se define $M_{i}:=p_{i} Y_{i}=\left(\frac{\gamma_{i}}{\gamma_{T}}\right) C_{T}+V_{i}^{*}$ donde la igualdad viene dada por la función -"de demanda"-(2). De las condiciones de primer orden para el productor del bien $i$, es fácil derivar que

$$
\frac{w_{n} L_{n, i}}{\theta_{i}}=p_{i} Y_{i}=M_{i}
$$

en donde la última igualdad se da porque, en equilibrio, la cantidad ofrecida debe igualar a la demandada. De (10) y la relación anterior se tiene que

$$
L_{c, i}=\frac{\delta_{i}}{w_{c}} M_{i}
$$

Esta relación crea una dependiencia entre la cantidad de trabajo calificado deseado y los aumentos en la demanda, que se reflejan en $M_{i}$. Para determinar el salario del trabajo calificado, se agrega para todos los sectores las funciones de demanda de trabajo a un mismo salario y se iguala la cantidad deseada de trabajo a la dotación de trabajo. Esto es, se agrega el $L_{c}$ encontrado en la relación anterior, manteniendo el salario $w_{c}$ constante, para obtener que

$$
w_{c}=\frac{\sum_{i=1}^{N}\left(\delta_{i} M_{i}\right)}{\overline{L_{c}}}
$$

en donde $\overline{L_{c}}$ es la dotación de trabajo calificado en la economía. La relación anterior determina el salario de equilibrio para el trabajo calificado. Note que esta relación depende de la demanda en cada uno de los sectores $i$. Sustituyendo este salario en la demanda de trabajo, se encuentra la cantidad de trabajo calificado de equilibrio para cada sector

$$
L_{c, i}=\left(\frac{\delta_{i} M_{i}}{\sum_{i=1}^{N} \delta_{i} M_{i}}\right) \overline{L_{c}}
$$

Utilizando nuevamente que $\frac{w_{n} L_{n, i}}{\theta_{i}}=M_{i}$ se obtiene que

$$
L_{n, i}=\frac{\theta_{i}}{w_{n}} M_{i}
$$


$y$ de (9) y (16), junto con (25) se tiene que

$$
\begin{aligned}
K_{i} & =\frac{\sigma_{i}}{r} M_{i} \\
S_{i} & =\frac{\psi_{i}}{H_{i}} M_{i}
\end{aligned}
$$

Las relaciones anteriores determinan las cantidades de trabajo no calificado, capital y tierra de equilibrio. Se puede notar que aumentos en la demanda por el bien $i$, que se reflejan en $M_{i}$, producen incrementos en las cantidades de factores de equilibrio; sustituyendo en la función de producción las cantidades de factores que se acaban de calcular e igualando a la demanda (2) se obtiene el precio del bien $i$

$$
p_{i}=R_{i}\left(\frac{\sum_{i=1}^{N}\left(\delta_{i} M_{i}\right)}{\delta_{i} \bar{L}_{c}}\right)^{\delta_{i}}
$$

en donde

$$
R_{i}=\frac{1}{A_{i}}\left(\frac{w_{n}}{\theta_{i}}\right)^{\theta_{i}}\left(\frac{H_{i}}{\psi_{i}}\right)^{\psi_{i}}\left(\frac{r}{\sigma_{i}}\right)^{\sigma_{i}}
$$

Note que el precio del bien $i$ depende de la demanda de todos los productos $i$ y no solamente de su demanda particular. Para determinar $C_{T}$ note que, utilizando la restricción presupuestaria, en equilibrio con $I_{j}=0$, (1) y suponiendo que $\gamma_{T}+\sum_{i=1}^{N} \gamma_{i}=1$, se obtiene que

$$
C_{T}=\gamma_{T}\left(r B_{t}+\overline{P I B}\right)
$$

De las condiciones de primer orden para cada uno de los sectores se deriva que los beneficios son cero. Utilizando esto se tiene que

$$
C_{T}=\gamma_{T}\left[r\left(B_{t}+K^{t o t}\right)+w_{n} L_{n}^{t o t}+w_{c} \overline{L_{c}}+r p_{S} S^{t o t}\right]
$$

en donde el superíndice tot se refiere al empleado en el total de la economía.

\section{Aumento en la demanda de los turistas}

Ahora se supone un aumento en la demanda por parte de los turistas en un bien particular $i$. Esto es un aumento en $V_{i}^{*}$ (producto, por ejemplo, de un incremento en el ingreso externo) que se refleja en un alza de $M_{i}$ (suponiendo constante $C_{T}$ ). Por la relación (28) se evidencia un aumento en el precio de todos los bienes $p_{i}$. Y un aumento en la producción en el sector $i$. De la ecuación de demanda (2), tomando logaritmos y diferenciando se obtiene que

$$
\widehat{Y}_{l}=\widehat{M}_{l}-\widehat{p}_{l}
$$

Aquí se debe notar que la cantidad $Y_{i}$, depende tanto de $M_{i}$, como del precio; así un aumento en $M_{i}$ podría disminuir el nivel de producción si el aumento en el precio, provocado por este aumento en $M_{i}$, es lo suficientemente grande. Podemos notar que este no es el caso, ya que de la ecuación del precio (28) se obtiene que

$$
\widehat{p_{\imath}}=\frac{\delta_{i}^{2} M_{i}}{\sum_{i=1}^{N}\left(\delta_{i} M_{i}\right)} \widehat{M}_{\imath}
$$

y como $\delta_{i}<1$, el cambio porcentual en el precio es menor al cambio porcentual en $M_{i}$, y se tiene un aumento en la producción.

Con el aumento en el precio, se eleva el valor del producto marginal de todos los factores que emplea el sector $i$, esto induce a que se incremente la cantidad contratada de estos factores en 
la región $i$. Con esto crece la demanda por trabajo calificado en este sector, lo cual se refleja en un aumento del salario de este tipo de trabajadores, según la relación (23). El alza del salario y la mayor contratación de trabajo calificado en el sector $i$ provocan que disminuya la cantidad de trabajo calificado en los otros sectores no transables. Note que según (24), (25), (26) y (27) solamente varía la cantidad de trabajo calificado, y se mantiene la contratación de los otros factores, esto suponiendo (por el momento) $C_{T}$ constante.

El decrecimiento en la contratación de trabajo calificado en los otros sectores se refleja en una disminución de la oferta en estos sectores y en el aumento en el precio de esos otros bienes. Es por esto que el precio del bien $j$ depende de la demanda del bien $i$, como se observaba anteriormente.

Por otra parte, la producción de transables se contrae en la zona donde se dio el aumento en la demanda. Esto es así por el desplazamiento de la tierra hacia la producción del bien $i$ cuya demanda se eleva; pero, en las otras zonas, no se presenta cambio en la producción $T$, ya que en los sectores no transables solamente cambia la contratación del trabajo calificado, no la contratación de tierra. Entonces, se espera que ante un aumento en la demanda de los turistas por un solo bien no transable, se eleve el precio de todos los bienes no transables, crezca la producción de no transable en el sector que enfrenta la mayor demanda, aumente el salario del trabajo calificado y disminuya la producción $Y_{j}$ de los otros bienes no transables. Además se reduce la producción de transable en la zona donde se produce el bien que sufre la perturbación externa, y no cambia en las demás zonas.

En la región que enfrenta el aumento de demanda por parte de los turistas, se da un crecimiento de la producción del bien no transable; pero a la vez, una disminución de la producción del bien transable, quedando indefinido el resultado total sobre la producción. Se analiza seguidamente, entonces, lo que sucede con la producción total en dicha región.

El PIB de la región $i$ está dado por

$$
P I B_{i}=Y_{T, i}+p_{i} Y_{N, i}
$$

de (27) se obtiene que

$$
p_{i} Y_{i}=\frac{H_{i}}{\psi_{i}} S_{i}
$$

De forma similar se puede expresar la producción de transables por

$$
Y_{T, i}=\frac{H_{i}}{\psi_{T}} S_{T}
$$

Con esto se puede expresar el cambio en la producción de la región $i$ como

$$
d P I B_{i}=\frac{H_{i}}{\psi_{i}} d S_{i}+\frac{H_{i}}{\psi_{T}} d S_{T}
$$

y recordando que $d S_{T}=-d S_{i}$ ya que la dotación de tierra está dada, se tiene que

$$
d P I B_{i}=H_{i}\left(\frac{\psi_{T}-\psi_{i}}{\psi_{T} \psi_{i}}\right) d S_{i}
$$

La relación anterior dice que el efecto predominante sobre la producción depende de la intensidad con la que se use la tierra en el sector $i$ relativo al sector transable. Si se supone que en esta zona el sector transable es intensivo en tierra, es decir $\psi_{T}>\psi_{i}$, se tiene que el efecto predominante sería el del sector no transable. De ser así, en la región en la que se da el aumento en la demanda se incrementaría la producción. 
En las demás regiones, como se indicaba, la producción de transables no se ve afectada, y se da una disminución en la producción del bien no transable $Y_{j}$, debido a que se expulsa trabajo calificado; pero junto a esta disminución en $Y_{j}$ se da un aumento en el precio, por lo que se requiere determinar qué sucede con $p_{j} Y_{j}$.

Por la condición de cero beneficios (derivada de las condiciones de primer orden) se tiene que

$$
p_{j} Y_{j}=w_{n} L_{n, j}+w_{c} L_{c, j}+r K_{j}+r p_{s} S_{j}
$$

De la expresión anterior solo están variando $w_{c}$ y $L_{c}$; pero anteriormente se había derivado que $w_{c} L_{c, j}=\delta_{j} M_{j}$, como se supone que no hay cambio en $C_{T}$, no hay variación en $M_{j}$, entonces no cambia $w_{c} L_{c, j} y$ no hay variación en la producción. Note que en este caso particular, la disminución en la cantidad de bienes $Y_{j}$ se ve compensada por el aumento en el precio, dejando el valor de la producción sin cambio.

Así, en el sector que se da la perturbación, el valor de la producción varía, pero no ocurre así en los otros sectores. De esta manera lo que sucede en el sector que experimenta el aumento en la demanda determina lo que ocurre con el PIB total de la economía. Si se asume que $\psi_{T}>\psi_{i}$ se tendría un aumento en el PIB de la economía; en caso contrario se daría una disminución.

A lo largo de este análisis se ha asumido que $C_{T}$ se mantiene constante. Note que si dicho consumo varía, se vería modificada la demanda por todos los bienes y no solamente la demanda por no transables en la zona particular $i$. De aumentar, estaría incrementando la demanda por todos los bienes no transables, por lo que se esperaría un alza en el valor de la producción, también en los otros sectores no transables; en lugar de mantenerse constante como se acaba de indicar.

De la relación (30) se tiene que

$$
C_{T}=\gamma_{T}\left[r\left(B_{t}+K^{t o t}\right)+w_{n} L_{n}^{t o t}+w_{c} \overline{L_{c}}+r p_{S} S^{t o t}\right]
$$

Nótese que el cambio en el total de capital se compensa con el cambio en los activos $B$, dejando en el estado estacionario, la riqueza $r\left(B_{t}+K^{t o t}\right)$ constante. La remuneración a la tierra $r p_{s} S^{t o t}$ tampoco sufre variación; pero aumenta tanto $w_{c}$ como $L_{n}$. Esto eleva el consumo $C_{T}$. Como se observa, este incremento en el consumo de no transables (proveniente de un aumento en el ingreso) provoca un efecto multiplicador en la economía. El crecimiento en $C_{T}$ se refleja en un alza de la demanda por todos los bienes no transables $i$, llevando a un aumento de sus precios, su producción y del salario del trabajo calificado. Y en este caso, sí se da un desplazamiento de la tierra en todos los sectores, por lo que se contrae la producción del bien transable en todas las regiones.

Note de la relación (30) anterior, que el cambio en $C_{T}$ depende del cambio en $w_{c} y \mathrm{~L}_{\mathrm{N}}{ }^{\text {tot }}$. Utilizando (25) y (23) se tiene que

$$
w_{n} L_{n}^{\text {tot }}+w_{c} \overline{L_{c}}=\sum_{i=1}^{N}\left[\left(\theta_{i}+\delta_{i}\right) M_{i}\right]
$$

Entonces el cambio en el consumo de transable se puede expresar como

$$
d C_{T}=\gamma_{T} \sum_{i=1}^{N}\left[\left(\theta_{i}+\delta_{i}\right) M_{i}\right]
$$

Si se supone un aumento en $V_{i}^{*}$ pero se mantienen constantes $V_{m}{ }^{*}$ para $m \neq i$, y se usa que $M_{i}=\frac{\gamma_{i}}{\gamma_{T}} C_{T}+V_{i}^{*}$ la relación anterior puede reescribirse de la siguiente manera

$$
d C_{T}=\left(\frac{\gamma_{T}\left(\theta_{i}+\delta_{i}\right)}{1-\sum_{j=1}^{N}\left(\theta_{j}+\delta_{j}\right) \gamma_{j}}\right)
$$


Entonces note que para tener un resultado estable se necesita que $\sum_{j=1}^{N}\left(\theta_{j}+\delta_{j}\right) \gamma_{j}$; pero se había supuesto que $0<\gamma_{T}<1,0<\gamma_{\mathrm{i}}<1, \theta_{\mathrm{j}}+\delta_{\mathrm{j}}<1$ y que $\sum_{i=1}^{N} \gamma_{i}=1-\gamma_{T}$, entonces se tiene que efectivamente $\sum_{j=1}^{N}\left(\theta_{j}+\delta_{j}\right) \gamma_{j}<1$.

Además note que el $C_{T}$ aumenta debido al crecimiento en $L_{n}{ }^{\text {tot }}$ y en $w_{c}$. Entonces, para que no se eleve $C_{T}$, deberían ser los no residentes los dueños del factor trabajo. De ser ese el caso, se espera una disminución de la utilidad de los residentes, ya que no disfrutan del aumento en el ingreso, pero se enfrentan al incremento en el precio de los bienes no transables.

Luego la relación (30), utilizando (1), puede rescribirse como

$$
C_{T}+\sum_{i=1}^{N} p_{i} C_{i}=r\left(B_{t}+K^{t o t}\right)+w_{n} L_{n}^{t o t}+w_{c} \overline{L_{c}}+r p_{s} S^{t o t}
$$

Esta relación dice que el gasto iguala al ingreso. Diferenciando esta relación, la función de utilidad instantánea y haciendo uso de (1),(23) y (25) , se tiene que la utilidad instantánea aumenta $\mathrm{si}^{6}$

$$
\sum_{i=1}^{N}\left(\theta_{i}+\delta_{i}\right) d M_{i}-\sum_{i=1}^{N} C_{i} d p_{i}>0
$$

El primer miembro de la resta anterior representa el cambio total en el gasto (ingreso); mientras que el segundo, el cambio en el gasto debido al aumento en los precios. La diferencia se interpreta como el cambio en el gasto debido a las variaciones en las cantidades consumidas y esto iguala al cambio en la utilidad instantánea (dividida entre la utilidad marginal de $C_{T}$, pero este componente es irrelevante para la desigualdad).

Utilizando (28), la relación anterior es equivalente a

$$
\sum_{i=1}^{N}\left(\theta_{i}+\delta_{i}\right) d M_{i}-\frac{\sum_{i=1}^{N}\left(\delta_{i}^{2} p_{i}\right) d M_{i}}{\sum_{i=1}^{N} \delta_{i} M_{i}}>0
$$

De cumplirse lo anterior la utilidad aumentaría, en caso contrario, el efecto del incremento del precio predominaría sobre el aumento del ingreso, disminuyendo el nivel de utilidad.

En este modelo se tiene que el aumento en la demanda de uno de los bienes no transables $i$ eleva la producción, no solo de este bien, si no de todos los demás bienes no transables; pero a la vez, disminuye la producción del bien transable en todas las regiones. El incremento en la producción de todos los bienes no transables se debe a un efecto multiplicador. El aumento en la demanda del bien no transable $i$ eleva el ingreso, lo cual se refleja en una mayor demanda por todos los bienes y esto es lo que provoca el aumento en la producción de los demás bienes $i$.

Adicionalmente se espera un crecimiento en el salario del trabajo calificado, ya que el aumento en la producción de los bienes no transables, se refleja en un incremento en la demanda por este factor y, como se asume que no es móvil a nivel internacional, se da un alza en el salario $w_{c}$. Además el resultado sobre el empleo y el acervo de capital totales en la economía dependen de las intensidades con las que se usen los factores en cada tipo de bien. Así, en general, no se puede decir que un

$6 \quad$ Es pertinente recordar que si se diferencia la función de utilidad se tiene que

$$
d U=u_{C_{T}}^{T} d C_{T}+u_{C_{N}}^{N} d C_{N}
$$

Entonces la utilidad aumenta si $u_{C_{T}}^{T} d C_{T}+u_{C_{N}}^{N} d C_{N}>0$. Como la utilidad marginal de $C_{T}$ es positiva y distinta de cero, se divide entre esta la relación anterior y se utiliza el hecho de que el precio iguala a la tasa marginal de sustitución, para obtener que la utilidad instantánea aumenta si

$$
d C_{T}+p d C_{N}>0
$$

Y una expresión para la relación anterior se puede obtener al diferenciar la relación de gasto=ingreso 
aumento del turismo eleve el total de contratación, ya que trabajo es absorbido por este sector, pero despedido del sector transable $T$. El resultado final depende de cuál de los sectores use más intensivamente el trabajo y el capital.

\section{Regiones especializadas en la producción de no transables y el precio de la tierra}

En el modelo anterior el precio de la tierra queda determinado en el sector no transable y no se ajusta ante aumentos en la demanda de los turistas. En esta sección se presenta un caso en el cual el aumento en el turismo puede elevar el precio de la tierra de algunas regiones.

En este caso, se supone, que existen ciertas regiones $j$, en las cuales, solamente se produce el bien no transable. En este caso las relaciones (5)-(12) siguen siendo válidas, junto con las relaciones (23)-(26).

Si se supone que $p_{s, j, t}=p_{s, j, t+1}$, de la relación (12), se obtiene que

$$
r p_{s, j, t}=\frac{\psi_{j}}{\theta_{j}} \frac{w_{n} L_{n, j}}{\overline{S_{J}}}
$$

En la relación anterior se toma en cuenta que el sector no transable ocupará toda la tierra de la región $\overline{S_{l}}$, ya que solamente se produce dicho bien. De la relación anterior (32) y (25) se obtiene el precio de equilibrio de la tierra en las regiones $j$, donde solo se produce el bien no transable. Dicho precio de equilibrio viene dado por

$$
p_{s, j}=\frac{\psi_{j}}{r} \frac{M_{j}}{\overline{S_{J}}}
$$

Se puede notar, que aumentos en $M_{j}$, derivados de incrementos en la demanda de los turistas, tienden a elevar el precio de la tierra en las regiones $j$, donde toda la tierra es empleada en la producción del bien no transable. Entonces, en las zonas especializadas, es de esperar que los aumentos del turismo provoquen un alza en el precio de la tierra.

\section{CONCLUSIONES}

La motivación del estudio era dar respuesta a las siguientes preguntas: ¿Cuál es el posible resultado sobre la producción interna, de largo plazo, de un aumento del gasto de los turistas en una economía como la costarricense?; ¿en el largo plazo, qué sectores pueden ver aumentada su producción y qué sectores pueden verla disminuida ante un aumento del gasto de los turistas en una economía como la costarricense? y ¿cuál es el posible resultado sobre los precios relativos, de largo plazo, de un aumento del gasto de los turistas en una economía como la costarricense?

Se ha visto que la producción interna puede aumentar o disminuir. El resultado depende de la intensidad con la que se usen los factores en los diferentes sectores. El principal efecto, que determina este cambio en la producción, es el cambio en el total de factores que se contratan en la economía. En el tanto los diferentes sectores compartan algún factor que no sea móvil internacionalmente, al expandirse un sector, el otro se contrae. El sector que se expande contrata más de los factores móviles internacionalmente, mientras que el que se contrae los despide. Como se supone pleno empleo para los factores no móviles a nivel internacional, estos no varían. Entonces, el total de factores utilizados en la economía puede disminuir si es mayor la cantidad que se expulsa del sector que se contrae, que la que se contrata en el sector que se expande. Así, si el sector que se contrae utiliza de forma muy intensiva (relativa a los factores no móviles) los factores móviles a nivel internacional; al despedir una unidad de factor no móvil, a la vez despide "mucho" factor móvil. El sector que se expande, cuando no es intensivo en esos factores móviles a nivel internacional, al contratar, no emplea a todos los factores que se expulsaron del sector que se contrae, ya que este es menos intensivo en dichos factores. Esta 
combinación puede disminuir la producción; pero junto con lo anterior, debe notarse que, a pesar del descenso en la producción, el ingreso podría no disminuir. Esto se debe a que el capital que es expulsado, puede ser reemplazado por activos externos, lo cual aumenta el ingreso.

Por otra parte, el sector que sufre la perturbación externa, tiende a expandirse. Los sectores que compartan el uso de factores no móviles a nivel internacional con el sector que se expande tienden a contraerse, o al menos, existe una fuerza que aplica en este sentido. Esto se da porque el sector que se expande contrata más de estos factores no móviles a expensas de los otros sectores que también los utilizan; pero, debe notarse, que existe otro efecto que puede contrarrestar este fenómeno: si la perturbación externa genera un aumento en el ingreso, se elevaría la demanda por todos los productos y se tiene un incentivo para expandir los demás sectores no transables.

Por último, dependiendo de la cantidad de factores móviles, no móviles y específicos a cada sector, el precio de los bienes no transables puede mantenerse o aumentar. En particular, la movilidad internacional de los factores tiende a disminuir la apreciación real; esto ya que ante el aumento en la demanda, se da un incentivo para contratar más factores, si estos son móviles a nivel internacional, pueden aumentarse sin modificar su precio (ya que sus precios estarían dados a nivel externo); esto lleva a que, ante aumentos en la demanda, se tienda a expandir la oferta en lugar de aumentar los precios.

Respecto al bienestar, en general, se puede concluir que existen dos efectos contrapuestos: el aumento del ingreso y el incremento en el precio. La mayor demanda por parte de los turistas no tiende a depreciar el ingreso de los nacionales ${ }^{7}$. De elevarse el ingreso, se generaría un efecto que tiende a aumentar el bienestar.; pero, el aumento en la demanda de los turistas no tiende a reducir el precio de los no transables ${ }^{8}$. De presentarse un alza en el precio, sería un efecto que tiende a disminuir el bienestar. Cuál de estos dos efectos predomina, y cuáles son sus repercusiones sobre la distribución del ingreso y la desigualdad, son cuestiones empíricas abiertas a investigación.

\section{AGRADECIMIENTOS}

Me gustría agradecer a Anabelle Ulate, Juan Rafael Vargas, José Antonio Cordero, Juan Muñoz, Marlon Yong y a un referee anónimo por sus valiosos comentarios. Todos los errores son mi responsabilidad.

\section{REFERENCIAS}

Balassa, B. (1964). "The Purchasing Power Parity Doctrine: A Reappraisal”. Journal of Political Economy, Vol72. Issue.6. December.

Barquero, Jorge y Vargas, Juan Carlos. (2004). "La Migración Internacional en Costa Rica: Estado Actual y Consecuencias". En: Evolución Demográfica de Costa Rica y su Impacto en los Sistemas de Salud y de Pensiones. San José, Academia de Centroamérica, 55-88.

Bryden, J.M.(1973).Tourism and Development: A Case Study of the Commonwealth Caribbean. Cambridge University Press.

Copeland, Brian. (1991). "Tourism, Welfare and De-industrialization in a Small Open Economy". Económica, New Series,Vol.58, No.232, November.

Corden, W.M. (1984). "Booming Sector and Dutch Disease Economics: Survey and Consolidation". Oxford Economic Papers, vol 36.

Corden, W.M. and Neary, J.P. (1982). "Booming sector and De-industrialization in a Small Open Economy”. Economic Journal,92, 825-48.

Es decir, lo aumenta o lo deja sin cambio.

8 Es decir, lo aumenta o lo deja sin cambio. 
Cordero José Antonio.(2000). "El Crecimiento Económico y la Inversión: el Caso de Costa Rica" en Ulate Anabelle,(ed.) Crecimiento, Empleo y Equidad: los Desafíos de las Reformas de Finales del siglo XX en Costa Rica, CEPAL-Editorial de la Universidad de Costa Rica, San José.

Chao, Chi-Chur; Hazari, Bharat; Laffargue, Jean-Pierre; Sgro, Pasquale and Yu, Eden.(2005). "Tourism, Jobs, Capital Accumulation and the Economy: A Dynamic Analysis".Paris-Jourdan Sciences Economiques, Working paper $\mathrm{N}^{\circ}$ 2005-16.

Gaceta. (1985). Ley de Incentivos para el DesarrolloTurístico, No. 143 del 30 de julio de 1985.

Latimer,H.(1986). "Developing Island Economies: Tourism v Agriculture". Tourism Management, Vol.6.

Luzzi, G.F. and Fluckiger, Y. (2003). “Tourism and International Trade. Introduction”. Pacific Economic Review. 8:3,239-243.

Nowak, Jean-Jacques and Sahli, Mondher.(2003). "Tourism, Trade and Domestic Welfare". Pacific Economic Review, Vol 8, №3.

Obstfeld, Maurice and Rogoff, Kenneth. (1996). Foundations of International Macroeconomics. Third Printing. The MIT Press. Cambridge, Massachusetts.

Porto, Guido. (2003). "Using Survey Data to Assess the Distributional Effects of Trade Policy". The World Bank, Development Research Group, WPS3137.

Rodríguez-Clare, Andrés; Saénz, Manrique y Trejos, Alberto. (2004). "Análisis del Crecimiento Económico en Costa Rica, 1950-2000" en Agosín Manuel, Machado Roberto y Nazal Paulina eds. Pequeñas Economías, Grandes Desafíos. BID,Washington DC. Capítulo VII.

Rojas, Luis Diego. (2008). "Modelos Macroeconómicos del Turismo y Evidencia para Costa Rica". Instituto de Investigaciones en Ciencias Económicas, Universidad de Costa Rica.

Rojas, Luis Diego. (2009). "Evolución e importancia del turismo en Costa Rica”. Informe Final, Quinto Informe Estado de La Nación en Desarrollo Humano Sostenible.

Samuelson, Paul A. (1964). "Theoretical Notes on Trade Problems". The Review of Economics and Statistics. Vol. 46, Issue 2, May.

Tyrakowski, K. (1986). "The Role of Tourism in Land Utilization Conflicts on the Spanish Mediterranean Coast". Geojournal, Vol.13.

Ulate, Anabelle y Rojas, Luis Diego.(2006)."Efectos Redistributivos de la Política Comercial". Presentado en el Simposio: Costa Rica a la luz de la Encuesta Nacional de Ingresos y Gastos de los Hogares, INEC, San José, Costa Rica.

Ulate, Anabelle y Rojas, Luis Diego. (2007). "Tipo de Cambio Real y Turismo: ¿Economía de una Enfermedad Tica?". Instituto de Investigaciones en Ciencias Económicas, Universidad de Costa Rica.

Weaver, D. (1988). "The Evolution of a Plantation Tourism Landscape on Caribbean Island of Antigua". Tiddschrift voor Economische en Sociale Geographic, Vol.79.

Yong, Marlon; Rojas, Luis Diego; Hernandez, Allan y Maroto, Mauricio. (2009). "Medición del Impacto de la Ley 6990 del Desarrollo Turístico en la Economía Nacional". Informe Final, Instituto de Investigaciones en Ciencias Económicas, Universidad de Costa Rica. 